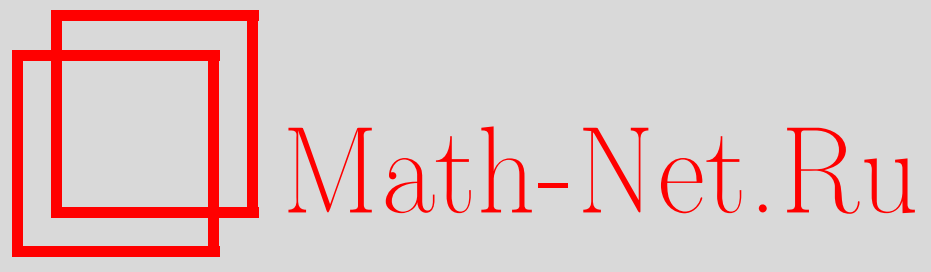

Л. В. Жижиашвили, О некоторых интегральных свойствах многомерного оператора Гильберта, Матем. заметки, 2006, том 80, выпуск 2, 179-186

DOI: https://doi.org/10.4213/mzm2797

Использование Общероссийского математического портала Math-Net.Ru подразумевает, что вы прочитали и согласны с пользовательским соглашением http://www . mathnet.ru/rus/agreement

Параметры загрузки:

IP : 3.80 .253 .173

26 апреля 2023 г., 16:06:54

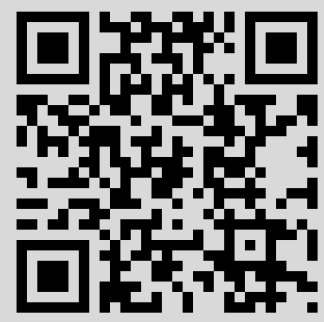




\section{О НЕКОТОРЫХ ИНТЕГРАЛЬНЫХ СВОЙСТВАХ МНОГОМЕРНОГО ОПЕРАТОРА ГИЛЬБЕРТА}

\section{Л. В. Жижиашвили}

В настоящей статье исследуется вопрос об интегральных свойствах многомерных преобразований Гильберта.

Библиография 15 названий.

Посвящается 100-летию со дня рождения С. М. Никольского.

1. Обозначения и определения. В дальнейшем $\mathbb{R}^{n}, n \geqslant 1$, будет обозначать $n$-мерное евклидово пространство с обычными линейными операциями; при этом считается, что $\left.\mathbb{R}^{1} \equiv \mathbb{R}=\right]-\infty,+\infty\left[\right.$. Точки пространства $\mathbb{R}^{n}$ обозначаются через $x=\left(x_{1}, x_{2}, \ldots, x_{n}\right), \quad s=\left(s_{1}, s_{2}, \ldots, s_{n}\right), t=\left(t_{1}, t_{2}, \ldots, t_{n}\right), \ldots$ Мы будем пользоваться некоторыми обозначениями из работ [1], [2]. Пусть $M=\{1,2, \ldots, n\}$, а $B$ его произвольное подмножество. Считаем, что $B^{\prime}=M \backslash B,|B|$ - число элементов множества $M$. Если $x \in \mathbb{R}^{n}$, то для произвольного непустого подмножества $B$ символом $x_{B}$ обозначим такую точку из $\mathbb{R}^{n}, n \geqslant 2$, координаты которой с индексами из множества $B$ совпадают с соответствующими координатами точки $x$, координаты же с индексами из множества $B^{\prime}$ - нули $\left(x_{M} \equiv x\right)$. Символом $\mathbb{R}^{n}(B)$ обозначим гиперплоскость, натянутую на координатные векторы, индексы которых составляют множество $B$, а через $d x-$ меру Лебега в $\mathbb{R}^{n}$.

Будем рассматривать вещественные функции $f: R^{n} \rightarrow R$. Если функция $f \in$ $L^{p}\left(\mathbb{R}^{n}\right)$ при некотором $p \in[1,+\infty[$, то

$$
\|f\|_{p}=\left\{\int_{\mathbb{R}^{n}}|f(x)|^{p} d x\right\}^{1 / p} .
$$

Если функция $f \in L\left(\mathbb{R}^{n}\right)$, то выражение

$$
\tilde{f}_{B}(x)=\frac{1}{\pi^{|B|}} \mathrm{v} \cdot \mathrm{p} \cdot \int_{\mathbb{R}^{n}(B)} f\left(x_{B^{\prime}}+s_{B}\right) \prod_{i \in B} \frac{1}{t_{i}-x_{i}} d t_{i}
$$

будем называть преобразованием (оператором) Гильберта $n$ переменных относительно тех переменных, индексы которых составляют множество $B$.

(C) Л.В. ЖижиАшвили, 2006 
ЗАмечаниЕ 1 . Если $n=1$ и функция $f \in L(\mathbb{R})$, то $\tilde{f}_{B}(x) \equiv \tilde{f}(x)$ - одномерное преобразование Гильберта, которое [3], [4], [5; с. 191], [6; с. 364], [7; с. 127-135] определено для почти всех $x \in \mathbb{R}$ равенством

$$
\tilde{f}(x)=\frac{1}{\pi} \lim _{\varepsilon \rightarrow 0} \int_{\varepsilon}^{\infty} \frac{f(x+t)-f(x-t)}{t} d t .
$$

В случае $n \geqslant 2$ при $2 \leqslant|B| \leqslant n$ преобразование $\tilde{f}_{B}$ функции $f \in L\left(\mathbb{R}^{n}\right)$ может не существовать почти всюду на $\mathbb{R}^{n}$. Поэтому при $n \geqslant 2$ определение (1.1) формальное.

Пусть $x \in \mathbb{R}, \varepsilon \in \mathbb{R}^{n}, \quad \varepsilon_{i}>0, i=\overline{1, n}$. Положим

$$
\mathbb{R}^{n}(B, x, \varepsilon)=\left\{t \in \mathbb{R}:\left|t_{i}-x_{i}\right| \geqslant \varepsilon_{i}, \quad i \in B\right\} .
$$

Для $x \in \mathbb{R}^{n}$ максимальный оператор по координатам, индексы которых составляют множество $B$, определяем (при $n \geqslant 2-$ формально) как

$$
F_{1}(x) \equiv F_{1}(x, f, B)=\sup _{\varepsilon}\left|\frac{1}{\pi^{|B|}} \int_{\mathbb{R}^{n}(B, x, \varepsilon)} f\left(x_{B^{\prime}}+t_{B}\right) \prod_{i \in B} \frac{1}{t_{i}-x_{i}} d t_{i}\right| .
$$

Заметим, что одномерный максимальный оператор $F_{1}$ конечен почти всюду на $\mathbb{R}$.

Далее, для функции $f \in L\left(\mathbb{R}^{n}\right)$ будем полагать, что

$$
\tilde{f}_{B}(x, \varepsilon)=\frac{1}{\pi^{|B|}} \int_{\mathbb{R}^{n}(B, x, \varepsilon)} f\left(x_{B^{\prime}}+t_{B}\right) \prod_{i \in B} \frac{d t_{i}}{t_{i}-x_{i}} .
$$

ЗАмечание 2. Ниже через $A, A(p), A(f), A(p, n), \ldots$ обозначаются, вообще говоря, различные положительные величины, зависящие лишь от указанных параметров.

2. Постановка вопроса. Хорошо известно ([8], [9], [5; с. 176], [6; с. 383], [7; c. $127-135],[10],\left[11 ;\right.$ с. 243-247]), что если функция $f \in L^{p}(\mathbb{R})$ при некотором $p \in$ ] $1,+\infty\left[\right.$, то $\tilde{f}$ существует почти всюду, $\tilde{f} \in L^{p}(\mathbb{R})$ и

$$
\|\tilde{f}\|_{p} \leqslant A(p)\|f\|_{p}
$$

причем соотношение (2.1) справедливо и для $F_{1}$, т.е.

$$
\left\|F_{1}\right\|_{p} \leqslant A(p)\|f\|_{p}
$$

Если же $f \in L(R)$, то $\tilde{f}$ существует почти всюду (см., например, [5; с. 191-192]), и для любого $p \in] 0,1[$ справедливо соотношение

$$
\int_{\mathbb{R}} \frac{|\tilde{f}(x)|^{p}}{1+x^{2}} d x<+\infty
$$

Добавим, что условие $f \in \bigcap_{p \geqslant 1} L^{p}(\mathbb{R})$ не гарантирует суммируемости на $\mathbb{R}$ преобразования $\tilde{f}$.

Кобер [12], [13] усилил сформулированное утверждение Титчмарша (см. (2.3)). В частности, в работе [12] он установил, что если функция $f \in L(\mathbb{R})$, то для интегрируемости $\tilde{f}$ на $\mathbb{R}$ необходимо условие

$$
\int_{\mathbb{R}} \tilde{f}(x) d x=0 .
$$


Если $n \geqslant 2$, то для $f \in L\left(\mathbb{R}^{n}\right)$ преобразования Гильберта $\tilde{f}_{B}$ с $2 \leqslant|B| \leqslant n$ могут не существовать почти всюду на $\mathbb{R}^{n}$ (см. $[1$; с. 132-151, 161-164], [2; с. 32-40]). Стало быть, вопрос об их интегральных свойствах некорректен.

Используя метод Сокол-Соколовского [14] и результаты Рисса [8], [9], можно заключить, что если функция $f \in L^{p}\left(\mathbb{R}^{n}\right)$ при некотором $\left.p \in\right] 1,+\infty[$, то все операторы $\tilde{f}_{B}$ с $1 \leqslant|B| \leqslant n$ существуют почти всюду на $\mathbb{R}^{n}$ и

$$
\left\|\tilde{f}_{B}\right\|_{p} \leqslant A(p, n)\|f\|_{p}
$$

Аналогично, опираясь на известные результаты (см., например, [6; с. 450], [10], [11; c. 243-247]) убеждаемся, что если функция $f \in L^{p}\left(\mathbb{R}^{n}\right)$ при некотором $\left.p \in\right] 1,+\infty[$, то для любого $B$ с $1 \leqslant|B| \leqslant n$ оператор $F_{1}$ из $(1.3)$ принадлежит классу $L^{p}\left(\mathbb{R}^{n}\right)$ и

$$
\left\|F_{1}\right\|_{p} \leqslant A(p, n)\|f\|_{p} .
$$

Возник вопрос: какой аналог имеет результат Кобера [12] (см. (2.4)) для многомерных операторов Гильберта?

Ниже этот вопрос решается.

\section{3. Об аналоге теоремы Кобера для многомерных операторов Гильбер-} та. Справедлива следующая

Теорема 1. Пусть функиия $f \in L\left(\mathbb{R}^{n}\right) \cap L^{2}\left(\mathbb{R}^{n}\right)$. Если для некоторого $B \subset M c$ $2 \leqslant|B| \leqslant n$ преобразование $\tilde{f}_{B} \in L\left(\mathbb{R}^{n}\right)$, mо

$$
\int_{\mathbb{R}^{n}} f(x) d x=0, \quad \int_{\mathbb{R}^{n}} \tilde{f}_{B}(x) d x=0 .
$$

ДокАзАтЕльство. Ниже будет установлено более общее утверждение, из которого, в частности, вытекают соотношения (3.1).

Используя условия сформулированной теоремы и обозначения (1.1), (1.4), будем иметь

$$
\lim _{\varepsilon_{B} \rightarrow 0+} \tilde{f}_{B}(x, \varepsilon)=\tilde{f}_{B}(x)
$$

почти всюду на $\mathbb{R}^{n}$ при любом $B$ с $1 \leqslant|B| \leqslant n$, причем $\tilde{f}_{B} \in L^{2}\left(\mathbb{R}^{n}\right), \quad \tilde{f}_{B}(\cdot, \varepsilon) \in$ $L^{2}\left(\mathbb{R}^{n}\right), \quad F_{1} \in L^{2}\left(\mathbb{R}^{n}\right)$, где $F_{1}$ определено в силу (1.4).

Для преобразования Фурье функции $f$ по тем координатам, индексы которых составляют множество $B$, будем использовать следующее обозначение:

$$
T_{B}(f)(x)=\left(\frac{1}{\sqrt{2 \pi}}\right)^{|B|} \int_{\mathbb{R}^{n}(B)} f\left(t_{B}+x_{B^{\prime}}\right) e^{i\left(t_{B} \cdot x_{B}\right)} \prod_{i \in B} d t_{i}, \quad 2 \leqslant|B| \leqslant n .
$$

Далее, если

$$
g_{\eta}(s)= \begin{cases}0, & |s| \leqslant \eta, \\ \sqrt{\frac{2}{\pi}} \frac{1}{s}, & \eta<|s|<+\infty, \quad \eta>0,\end{cases}
$$

то согласно (1.4) выражение $\tilde{f}_{B}(x, \varepsilon)$ можно представить следующим образом:

$$
\tilde{f}_{B, \varepsilon}(x) \equiv \tilde{f}_{B}(x, \varepsilon)=\left(\frac{1}{\sqrt{2 \pi}}\right)^{|B|} \int_{\mathbb{R}^{n}(B)} f\left(t_{B}+x_{B^{\prime}}\right) \prod_{i \in B} g_{\varepsilon_{i}}\left(t_{i}-x_{i}\right) d t_{i} .
$$


Используя хорошо известные результаты (см., например, [5; с. 119-122], [11; c. 245]), можно сделать следующее заключение:

$$
T_{B}\left(\tilde{f}_{B, \varepsilon}\right)(x)=T_{B}(f)(x) \prod_{i \in B} T\left(g_{\varepsilon_{i}}\right)\left(x_{i}\right),
$$

где $T(g)(x)$ - одномерное преобразование Фурье функции $g$.

Принимая во внимании условия теоремы 3.1 , согласно известной теореме Планшереля (см., например, [5; с. 92-102], [6; с. 374-375], [11; с. 25-26]) будем иметь

$$
\left\|T_{B}\left(\tilde{f}_{B}\right)-T_{B}\left(\tilde{f}_{B, \varepsilon}\right)\right\|_{2}=\left\|\tilde{f}_{B}-\tilde{f}_{B, \varepsilon}\right\|_{2} .
$$

Далее, предположим, что

$$
\varepsilon_{i}=\frac{1}{m_{i}}, \quad m_{i} \rightarrow \infty, \quad i \in B,
$$

и $m(B)=\left(m_{i_{1}}, m_{i_{2}}, \ldots, m_{i_{k}}\right)$ для $B=\left\{i_{1}, i_{2}, \ldots, i_{k}\right\}$. Тогда в силу (3.7) находим

$$
\lim _{m(B) \rightarrow \infty}\left\|T_{B}\left(\tilde{f}_{B}\right)-T_{B}\left(\tilde{f}_{B, \frac{1}{m}}\right)\right\|_{2}=0 .
$$

Согласно нашим предложениям $(m(B))-k$-кратная последовательность (так как $|B|=k)$. Опираясь на (3.8), можно выделить подпоследовательность, зависящую лишь от одного натурального индекса $\left(\nu_{1}(\alpha), \ldots, \nu_{k}(\alpha)\right)$ такую, что если в $T_{B}\left(\tilde{f}_{B}, 1 / m\right)(x)$ вместо $1 / m$ возьмем $1 / \nu(\alpha)$, то

$$
\lim _{\alpha \rightarrow \infty} T_{B}\left(\tilde{f}_{B}, \frac{1}{\nu(\alpha)}\right)(x)=T_{B}\left(\tilde{f}_{B}\right)(x)
$$

почти всюду на $\mathbb{R}^{n}$. Тогда, перейдя к пределу в равенстве (3.6), будем иметь

$$
T_{B}\left(\tilde{f}_{B}\right)(x)=(-1)^{|B|} T_{B}(f)(x) \prod_{i \in B} \operatorname{sign} x_{i}
$$

почти всюду на $\mathbb{R}^{n}(B)$. Следует добавить, что $x_{\nu}, \nu \in B^{\prime}$, фиксированы. Затем, легко видеть, что

$$
\begin{array}{llll}
(-i)^{|B|}=1 & \text { при } & |B|=4 k, & k \in \mathbb{N}, \\
(-i)^{|B|}=-1 & \text { при } & |B|=2 k-2, & k \in \mathbb{N}, \\
(-i)^{|B|}=i & \text { при } & |B|=4 k-1, & k \in \mathbb{N}, \\
(-i)^{|B|}=-i & \text { при } & |B|=4 k-3, & k \in \mathbb{N} .
\end{array}
$$

Далее, обозначим через $U$ подмножества $B$ с четным числом элементов. Будем также предполагать, что $V=B \backslash U$. Имеем

$$
\begin{array}{lll}
\prod_{i \in B} \operatorname{sign} x_{i}=1 & \text { при } \left.\quad x_{i} \in\right] 0,+\infty[, \quad i \in B, \\
\prod_{i \in B} \operatorname{sign} x_{i}=1 & \text { при } \left.\quad x_{i} \in\right]-\infty, 0\left[, \quad i \in U, \quad x_{j} \in\right] 0,+\infty[, \quad j \in V, \\
\prod_{i \in B} \operatorname{sign} x_{i}=-1 & \text { при } \left.\quad x_{i} \in\right] 0,+\infty\left[, \quad i \in U, \quad x_{j} \in\right]-\infty, 0[, \quad j \in V .
\end{array}
$$


В дальнейшем будем использовать следующие обозначения:

$$
\begin{aligned}
& H_{1} \equiv H_{1}(B)=\left\{x_{B}: x_{i} \in\right] 0,+\infty[, \quad i \in B, \quad|B|=4 k, \quad k \in \mathbb{N}\}, \\
& H_{2} \equiv H_{2}(B) \equiv H_{2}(B, U, V)=\left\{x_{B}: x_{i} \in\right]-\infty, 0[, \quad i \in U, \\
&\left.x_{j} \in\right] 0,+\infty[, \quad j \in V, \quad|B|=4 k, \quad k \in \mathbb{N}\}, \\
& H_{3} \equiv H_{3}(B)=\left\{x_{B}: x_{i} \in\right] 0,+\infty[, \quad i \in B, \quad|B|=4 k-2, \quad k \in \mathbb{N}\}, \\
& H_{4} \equiv H_{4}(B) \equiv H_{4}(B, U, V)=\left\{x_{B}: x_{i} \in\right]-\infty, 0[, \quad i \in U, \\
&\left.x_{j} \in\right] 0,+\infty[, \quad j \in V, \quad|B|=4 k-2, \quad k \in \mathbb{N}\}, \\
& H_{5} \equiv H_{5}(B)=\left\{x_{B}: x_{i} \in\right] 0,+\infty[, \quad i \in B, \quad|B|=4 k-1, \quad k \in \mathbb{N}\}, \\
& H_{6} \equiv H_{6}(B) \equiv H_{6}(B, U, V)=\left\{x_{B}: x_{i} \in\right]-\infty, 0[, \quad i \in U,\left.x_{j} \in\right] 0,+\infty[, \quad j \in V, \quad|B|=4 k-1, \quad k \in \mathbb{N}\}, \\
& H_{7} \equiv H_{7}(B)=\left\{x_{B}: x_{i} \in\right] 0,+\infty[, \quad i \in B, \quad|B|=4 k-3, \quad k \in \mathbb{N}\}, \\
& H_{8} \equiv H_{8}(B) \equiv H_{8}(B, U, V)=\left\{x_{B}: x_{i} \in\right]-\infty, 0[, \quad i \in U, \\
&\left.\quad x_{j} \in\right] 0,+\infty[, \quad j \in V, \quad|B|=4 k-3, \quad k \in \mathbb{N}\} .
\end{aligned}
$$

Если в множествах (3.17)-(3.24) предполагается $\left.\left.x_{i} \in\right]-\infty, 0\right], i \in U, x_{j} \in[0,+\infty[$, $j \in V, x_{i} \in[0,+\infty[, \quad i \in B$, а остальные предположения не меняются, то их будем обозначать через $\bar{H}_{s}, s=\overline{1,8}$.

Предположим, что

$$
\begin{aligned}
& \varphi\left(t_{B}, x_{B}\right)=\sum_{U \subset B} i^{|U|} \prod_{i \in U} \sin t_{i} x_{i} \prod_{j \in V} \cos t_{j} x_{j} \\
& \psi\left(t_{B}, x_{B}\right)=\sum_{V \subset B} i^{|V|} \prod_{i \in V} \sin t_{i} x_{i} \prod_{j \in U} \cos t_{j} x_{j}
\end{aligned}
$$

причем считаем, что

$$
\prod_{i \in U} \sin t_{i} x_{i}=1 \quad \text { при } \quad|U|=0, \quad \prod_{j \in V} \cos t_{j} x_{j}=1 \quad \text { для } \quad|V|=0 .
$$

Нетрудно проверить, что для $B \subset M$ с $2 \leqslant|B| \leqslant n$ каждое из выражений $\varphi$ и $\psi$ содержит $2^{|B|-1}$ слагаемых.

Затем, используя (3.25) и (3.26), будем иметь

$$
e^{i\left(t_{B} \cdot x_{B}\right)}=\varphi\left(t_{B}, x_{B}\right)+i \psi\left(t_{B}, x_{B}\right), \quad 2 \leqslant|B| \leqslant n .
$$

Вычисляя и приравнивая вещественные и мнимые части в равенстве (3.9) с использованием (3.10)-(3.27), находим

$$
\int_{\mathbb{R}^{n}(B)} \tilde{f}_{B}\left(t_{B}+x_{B^{\prime}}\right) \varphi\left(t_{B}, x_{B}\right) \prod_{i \in B} d t_{i}=\int_{\mathbb{R}^{n}(B)} f\left(t_{B}+x_{B^{\prime}}\right) \varphi\left(t_{B}, x_{B}\right) \prod_{i \in B} d t_{i}
$$

почти всюду на $H_{1}$ и $H_{2}$;

$$
\int_{\mathbb{R}^{n}(B)} \tilde{f}_{B}\left(t_{B}+x_{B^{\prime}}\right) \varphi\left(t_{B}, x_{B}\right) \prod_{i \in B} d t_{i}=-\int_{\mathbb{R}^{n}(B)} f\left(t_{B}+x_{B^{\prime}}\right) \varphi\left(t_{B}, x_{B}\right) \prod_{i \in B} d t_{i}
$$


почти всюду на $\mathrm{H}_{3}$ и $\mathrm{H}_{4}$;

$$
\int_{\mathbb{R}^{n}(B)} \tilde{f}_{B}\left(t_{B}+x_{B^{\prime}}\right) \varphi\left(t_{B}, x_{B}\right) \prod_{i \in B} d t_{i}=-\int_{\mathbb{R}^{n}(B)} f\left(t_{B}+x_{B^{\prime}}\right) \psi\left(t_{B}, x_{B}\right) \prod_{i \in B} d t_{i}
$$

почти всюду на $\mathrm{H}_{5}$ и $\mathrm{H}_{6}$;

$$
\int_{\mathbb{R}^{n}(B)} \tilde{f}_{B}\left(t_{B}+x_{B^{\prime}}\right) \varphi\left(t_{B}, x_{B}\right) \prod_{i \in B} d t_{i}=\int_{\mathbb{R}^{n}(B)} f\left(t_{B}+x_{B^{\prime}}\right) \psi\left(t_{B}, x_{B}\right) \prod_{i \in B} d t_{i}
$$

почти всюду на $H_{7}$ и $H_{8}$. Соотношения (3.28)-(3.31) справедливы для почти всех фиксированных $x_{\nu}, \nu \in B^{\prime}$. Учитывая, что по условию функции $f$ и $\tilde{f}_{B}$ принадлежат к классу $L\left(\mathbb{R}^{n}\right)$, интегрирование равенств (3.28)-(3.31) дает

$$
\int_{\mathbb{R}^{n}} \tilde{f}_{B}(t) \varphi\left(t_{B}, x_{B}\right) d t=\int_{\mathbb{R}^{n}} f(t) \varphi\left(t_{B}, x_{B}\right) d t
$$

почти всюду на $H_{1}$ и $H_{2}$;

$$
\int_{\mathbb{R}^{n}} \tilde{f}_{B}(t) \varphi\left(t_{B}, x_{B}\right) d t=-\int_{\mathbb{R}^{n}} f(t) \varphi\left(t_{B}, x_{B}\right) d t
$$

почти всюду на $\mathrm{H}_{3}$ и $H_{4}$;

$$
\int_{\mathbb{R}^{n}} \tilde{f}_{B}(t) \varphi\left(t_{B}, x_{B}\right) d t=-\int_{\mathbb{R}^{n}} f(t) \psi\left(t_{B}, x_{B}\right) d t
$$

почти всюду на $H_{5}$ и $H_{6}$;

$$
\int_{\mathbb{R}^{n}} \tilde{f}_{B}(t) \varphi\left(t_{B}, x_{B}\right) d t=\int_{\mathbb{R}^{n}} f(t) \psi\left(t_{B}, x_{B}\right) d t
$$

почти всюду на $H_{7}$ и $H_{8}$.

С другой стороны, имеем

$$
\|\tilde{f}\|_{1} \leqslant A\left(\tilde{f}_{B}\right), \quad\|f\|_{1} \leqslant A(f) .
$$

Тогда, опираясь на (3.32)-(3.35), приходим к заключению, что

$$
\int_{\mathbb{R}^{n}} \tilde{f}_{B}(t) \varphi\left(t_{B}, x_{B}\right) d t=\int_{\mathbb{R}^{n}} f(t) \varphi\left(t_{B}, x_{B}\right) d t
$$

всюду на $\bar{H}_{1}$ и $\bar{H}_{2}$;

$$
\int_{\mathbb{R}^{n}} \tilde{f}_{B}(t) \varphi\left(t_{B}, x_{B}\right) d t=-\int_{\mathbb{R}^{n}} f(t) \varphi\left(t_{B}, x_{B}\right) d t
$$

всюду на $\bar{H}_{3}$ и $\bar{H}_{4}$;

$$
\int_{\mathbb{R}^{n}} \tilde{f}_{B}(t) \varphi\left(t_{B}, x_{B}\right) d t=-\int_{\mathbb{R}^{n}} f(t) \psi\left(t_{B}, x_{B}\right) d t
$$

всюду на $\bar{H}_{5}$ и $\bar{H}_{6}$;

$$
\int_{\mathbb{R}^{n}} \tilde{f}_{B}(t) \varphi\left(t_{B}, x_{B}\right) d t=\int_{\mathbb{R}^{n}} f(t) \psi\left(t_{B}, x_{B}\right) d t
$$


всюду на множествах $\bar{H}_{7}$ и $\bar{H}_{8}$. Следует добавить, что при заданном $B$ с $2 \leqslant|B| \leqslant n$ число этих множеств (в силу их зависимости от $U$ и $V$ ) будет $2^{|B|+1}$. Стало быть, в (3.36)-(3.39) имеем $2^{|B|+1}$ равенств.

Таким образом, если в (3.36)-(3.39) положим $x_{i}=0, i \in B$, то убеждаемся в справедливости (3.1).

Теорема 1 доказана.

ЗАмечАниЕ 3. На самом деле, соотношения (3.25), (3.26) и (3.36)-(3.39) показывают, что было доказано более общее утверждение.

ЗАмЕчАниЕ 4. В том случае, когда $|B|=1$, справедливость теоремы 1 можно получить с применением соответствующего результата Кобера [12]. Следует добавить, что при $|B|=1$ требование $f \in L^{2}\left(\mathbb{R}^{n}\right)$ является лишним. Если же $2 \leqslant|B| \leqslant n$, то, как уже было сказано, $\tilde{f}_{B}$ в классе $L\left(\mathbb{R}^{n}\right)$ может не существовать почти всюду.

ЗАмечание 5 . При $1 \leqslant p \leqslant+\infty, 1 \leqslant q<+\infty, p \neq q$, не имеет место вложение пространств $L^{p}\left(\mathbb{R}^{n}\right)$ и $L^{q}\left(\mathbb{R}^{n}\right)$, поэтому предположение $f \in L^{2}\left(\mathbb{R}^{n}\right)$ в теореме 1 нельзя считать существенным ограничением.

ЗАмечАниЕ 6 . Пусть функция $f \in L^{p}\left(\mathbb{R}^{n}\right)$ при некотором $\left.p \in\right] 1,+\infty[$. Тогда, как уже было отмечено, оператор $\tilde{f}_{B}$ с $1 \leqslant|B| \leqslant n$ почти всюду существует на $\mathbb{R}^{n}$ и имеет место неравенство (2.1). Добавим, что почти всюду на $\mathbb{R}^{n}$ справедлива двойственная формула

$$
f(x)=\left(-\frac{1}{\pi}\right)^{|B|} \int_{\mathbb{R}^{n}(B)} \tilde{f}_{B}\left(t_{B}+x_{B^{\prime}}\right) \prod_{i \in B} \frac{d t_{i}}{t_{i}-x_{i}} .
$$

Добавим также, что (3.40) определяется как несобственный интеграл. В частности, почти всюду на $\mathbb{R}^{n}$

$$
\tilde{f}_{M}(x)=\frac{1}{\pi^{n}} \int_{\mathbb{R}^{n}} f(t) \prod_{i=1}^{n} \frac{d t_{i}}{t_{i}-x_{i}}
$$

и

$$
f(x)=\left(-\frac{1}{\pi}\right)^{n} \int_{\mathbb{R}^{n}} \tilde{f}_{M}(t) \prod_{i=1}^{n} \frac{d t_{i}}{t_{i}-x_{i}}
$$

почти для всех $x \in \mathbb{R}^{n}$.

ЗАмЕчАниЕ 7. Схема доказательства основной теоремы показывает, что теорема справедлива для $n$-кратных сингулярных операторов в том случае, когда ядром интеграла является прямое произведение простых ядер типа Кальдерона-Зигмунда [15].

\section{СПИСОК ЦИТИРОВАННОЙ ЛИТЕРАТУРЫ}

[1] L. Zhizhiashvili, Trigonometric Fourier Series and Their Conjugates, Kluwer Acad. Publ., Dordrecht-Boston-London, 1996.

[2] Л.В. ЖКжиашвили, Некоторые вопросы многомерного гармонического анализа, ТГУ, Тбилиси, 1996.

[3] A. Besicovitch, "Sur la nature des fonctions a carré sommable mésurables", Fund. Math., 4 (1923), 172-195. 
[4] A. Besicovitch, "On a general metric property of summable functions", J. Lond. Math. Soc., 1 (1926), 120-128.

[5] Е. Титчмарш, Введение в теорию интегралов Фуръе, Гостехиздат, М.-Л., 1948.

[6] А. Зигмунд, Тригонометрические рядь, т. 2, Мир, М., 1965.

[7] Дж. Гарнетт, Ограниченные аналитические функиии, Наука, М., 1984.

[8] M. Riesz, "Les fonctions conjuguées et les series de Fourier", Comp. Rend., 178 (1924), 1464-1467.

[9] M. Riesz, "Sur les fonctions conjuguées", Math. Z., 27 (1927), 218-244.

[10] A. Zygmund, Intégrales Singularières. Lecture Notes in Math., 204, Springer-Verlag, Berlin-Heidelberg-New York, 1971.

[11] И. Стейн, Г. Вейс, Введение в гармонический анализ на евклидовых пространствах, Мир, М., 1974.

[12] H. Kober, "A note on Hilbert's operator", Bull. Amer. Math. Soc., 48:1 (1942), 421-426.

[13] H. Kober, "A note on Hilbert transforms", J. Lond. Math. Soc., 18:2 (1943), 66-71.

[14] K. Sokol-Sokolowski, "On trigonometric series conjugate to Fourier series of two variables", Fund. Math., 34 (1947), 166-182.

[15] A. P. Calderon, A. Zygmund, "On the existence of certain singular integrals", Acta Math., 88:1-2 (1952), 85-139.

Л. В. Жижиашвили

Тбилисский государственный университет
Поступило

25.05.2005 